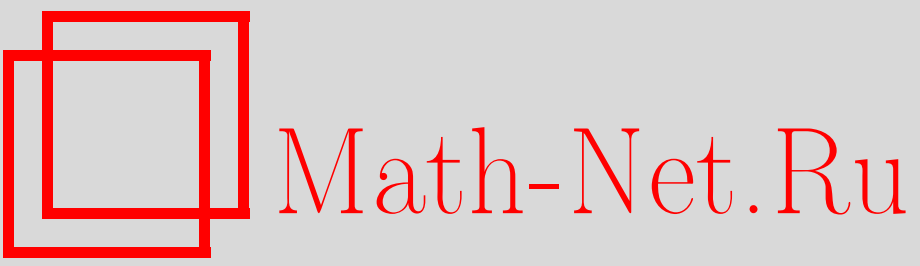

А. Я. Мальцев, Усреднение локальных теоретико-полевых скобок Пуассона, УМН, 1997, том 52, выпуск 2, 177-178

DOI: https://doi.org/10.4213/rm839

Использование Общероссийского математического портала Math-Net.Ru подразумевает, что вы прочитали и согласны с пользовательским соглашением

http://www.mathnet.ru/rus/agreement

Параметры загрузки:

IP: 54.172 .240 .79

26 апреля 2023 г., 17:41:46 


\title{
УСРЕДНЕНИЕ ЛОКАЛЬНЫХ ТЕОРЕТИКО-ПОЛЕВЫХ СКОБОК ПУАССОНА
}

\author{
А. Я. МАЛЬцЕВ
}

В работах [1]-[2] Б. А. Дубровиньм и С. П. Новиковьм была предложена процедура усреднения локальных теоретико-полевых гамильтоновых структур, дающая скобку Пуассона для уравнений Уизема, описьвающих эволюцию медленно промодулированных $m$-фазных решений систем нелинейньх уравнений [3], [4]. Опишем вкратце эту процедуру.

Пусть эволюционная система

$$
\varphi_{t}^{i}=Q^{i}\left(\boldsymbol{\varphi}, \boldsymbol{\varphi}_{x}, \ldots\right), \quad i=1, \ldots, n,
$$

является гамильтоновой относительно локальной теоретико-полевой скобки Пуассона вида:

$$
\left\{\varphi^{i}(x), \varphi^{j}(y)\right\}=\sum_{k \geqslant 0} B_{k}^{i j}\left(\varphi, \varphi_{x}, \ldots\right) \delta^{(k)}(x-y)
$$

(в сумме присутствует конечное число слагаемых), с гамильонианом:

$$
H=\int \mathscr{P}_{H}\left(\boldsymbol{\varphi}, \boldsymbol{\varphi}_{x}, \ldots\right) d x
$$

Пусть система (1) имеет $N$-параметрическое семейство (не считая сдвигов начальных фаз $\theta_{0}^{\alpha}$ ) $m$-фозных решений:

$$
\varphi^{i}(x, t)=\Phi^{i}\left(\mathbf{k}(\mathbf{U}) x+\boldsymbol{\omega}(\mathbf{U}) t+\boldsymbol{\theta}_{0}, \mathbf{U}\right),
$$

где $\mathbf{U}=\left(U^{1}, \ldots, U^{N}\right)$ - параметры семейства, $\Phi^{i}(\boldsymbol{\theta}, \mathbf{U})-2 \pi$-периодические функции $\boldsymbol{\theta}=$ $\left(\theta^{1}, \ldots, \theta^{m}\right)$, и $N$ коммутирующих между собой первых интегралов вида

$$
I^{\nu}=\int \mathscr{P}^{\nu}\left(\boldsymbol{\varphi}, \boldsymbol{\varphi}_{x}, \ldots\right) d x, \quad \nu=1, \ldots, N,
$$

попарные скобки Пуассона локальных плотностей которых имеют вид:

$$
\left\{\mathscr{P}^{\nu}\left(\boldsymbol{\varphi}, \boldsymbol{\varphi}_{x}, \ldots\right), \mathscr{P}^{\mu}\left(\boldsymbol{\varphi}, \boldsymbol{\varphi}_{y}, \ldots\right)\right\}=\sum_{k \geqslant 0} A_{k}^{\nu \mu}\left(\boldsymbol{\varphi}, \boldsymbol{\varphi}_{x}, \ldots\right) \delta^{(k)}(x-y),
$$

причем $A_{0}^{\nu \mu}=\partial_{x} Q^{\nu \mu}\left(\boldsymbol{\varphi}, \boldsymbol{\varphi}_{x}, \ldots\right)$.

Мы без нарушения общности будем считать, что значения параметров $U^{\nu}$ совпадают со значениями интегралов $I^{\nu}$ на соответствующих функциях семейств (4). При этом, потоки, порожденные в силу (2) интегралами $I^{\nu}$ (среди которых может быть и гамильтониан $H$ ), коммутируют между собой и с потоком (1) и оставляют семейство (4) инвариантным, порождая на нем линейную зависимость сдвига начальных фаз $\boldsymbol{\theta}_{0}$ от времен $\tau^{\nu}$, и оставляя неизменньми значения параметров $U^{\nu}$. T.e.

$$
\varphi^{i}\left(x, \tau^{1}, \ldots, \tau^{N}\right)=\Phi^{i}\left(\mathbf{k} x+\boldsymbol{\omega}^{(1)}(\mathbf{U}) \tau^{1}+\cdots+\boldsymbol{\omega}^{(N)} \tau^{N}+\boldsymbol{\theta}_{0}, \mathbf{U}\right)
$$

(

В этой ситуации скобку Дубровина-Новикова на пространстве полей $U^{\nu}(X)$ можно записать в виде

$$
\left\{U^{\nu}(X), U^{\mu}(Y)\right\}=\left\langle A_{1}^{\nu \mu}\right\rangle(\mathbf{U}(X)) \delta^{\prime}(X-Y)+\frac{\partial\left\langle Q^{\nu \mu}\right\rangle}{\partial U^{\lambda}} U_{X}^{\lambda} \delta(X-Y),
$$

где $\langle\ldots\rangle$ означает усреднение на семействе $m$-фразных решений $(1), U^{\nu}(X)$ - медленно меняющиеся функции координаты $x=X / \varepsilon, \varepsilon \rightarrow 0$.

Работа вьполнена при финансовой поддержке KFA Forschungszentrum Jülich GmbH и Российского фонда фундаментальных исследований (грант № 96-01-01623). 
Теория скобок (8) тесно связана с Римановой геометрией. В частности, если матрица $\left\langle A_{1}^{\nu \mu}\right\rangle$ невырождена, то (8) задает скобку Пуассона тогда и только тогда, когда величины $g_{\nu \tau}(\mathbf{U})$ и $\Gamma_{\mu \lambda}^{\nu}=-g_{\mu \tau} \partial\left\langle Q^{\tau \nu}\right\rangle / \partial U^{\lambda}$, где $g_{\nu \tau}\left\langle A_{1}^{\tau \mu}\right\rangle \equiv \delta_{\nu}^{\mu}$, задают на пространстве параметров $\mathbf{U}$ симметричную плоскую метрику и согласованную с ней симметричную связность, соответственно. Из тождества Лейбница для (8) вытекает при этом, что при заменах координат на пространстве параметров $\mathbf{U}$ величины $g_{\nu \tau}$ и $\Gamma_{\mu \lambda}^{\nu}$ преобразуются соответствующим образом. Теория скобок (8) с вырожденным тензором $\left\langle A_{1}^{\nu \mu}\right\rangle$ более сложна, но также имеет красивую геометрическую интерпретацию (см. [5]).

В [1]-[2], однако, отсутствовало доказательство тождества Якоби для полученной таким образом скобки (8), о чем сообщалось в [6]. Здесь мы сформулируем доказанные в [7] (с помощью процедуры ограничения по Дираку скобки Пуассона на подмногообразие в функциональном пространстве) теоремы о том, что (8) действительно однозначно задает скобку Пуассона на пространстве полей $U^{\nu}(X)$. Именно:

ТЕОрема 1. При сформулированных выше условиях, а также выполнении некоторых свойств регулярности (см. [7]), соответствующих общему положению:

1) скобка Дубровина-Новикова, определяемая формулой (8), удовлетворяет тождеству Якоби;

2) в силу (8) выполняются соотношения:

$$
\begin{gathered}
\left\{k^{\alpha}(\mathbf{U}(X)), U^{\nu}(Y)\right\}=\omega^{\alpha(\nu)}(\mathbf{U}(X)) \delta^{\prime}(X-Y)+\frac{\partial \omega^{\alpha(\nu)}(\mathbf{U}(X))}{\partial X} \delta(X-Y), \\
\left\{k^{\alpha}(\mathbf{U}(X)), k^{\beta}(\mathbf{U}(Y))\right\}=0, \quad \alpha, \beta=1, \ldots, m, \quad \nu=1, \ldots, N .
\end{gathered}
$$

Потоки, порожденнье интегралами $\int U^{\nu}(X) d X$ на пространстве полей $\mathbf{U}(X)$ в силу скобки (8), коммутируют между собой и дают уравнения Уизема для нелинейных систем, порожденных интегралами $I^{\nu}$ на пространстве полей $\varphi(x)$ в силу (2), соответств енно.

Теорема 2. Пусть у системы (1) имеется два несовпадающих набора интегралов вида (5), $\left\{I^{1}, \ldots, I^{N}\right\}$ и $\left\{\bar{I}^{1}, \ldots, \bar{I}^{N}\right\}$, обладающих всеми требуемьми свойствами. Тогда скобки Дубровина-Новикова, полученные с помощью этих наборов, совпадают, т.е. если на семействе (4): $U^{\nu}=I^{\nu}, \bar{U}^{\nu}=\bar{I}^{\nu}$, то скобка (8), полученная с помощью набора $\left\{I^{1}, \ldots, I^{N}\right\}$, после замены параметров на семействе $m$-фазных решений вида $U^{\nu}=U^{\nu}(\overline{\mathbf{U}})$ переходит в скобку, полученную с помощью набора $\left\{\bar{I}^{1}, \ldots, \bar{I}^{N}\right\}$.

Из теорем 1 и 2 вытекает также, что любой интеграл $I$ вида $(5)$, коммутирующий с гамильтонианом и со всеми $I^{\nu}$, при ограничении на семейство (4) дает плотность закона сохранения для систем, порожденных интегралами $\int U^{\nu}(X) d X$ в силу (8), а уравнения Уизема для нелинейной системы, порожденной $I$ в силу (2), дают коммутирующий поток к этим системам. Этот факт, как нетрудно видеть, следует из того, что интеграл $I$ можно включить в набор (5) вместо любого из имеющихся там интегралов $I^{\nu}$, и это не изменит скобки (8).

В заключение автор благодарит С.П. Новикова за постановку задачи и внимание к работе, а также В. Л. Алексеева, О. И. Мохова, М. В. Павлова и Е. В. Ферапонтова за плодотворные обсуждения.

\section{СПИСОК ЛИТЕРАТУРЫ}

[1] Дубровин Б.А., Новиков С.П. // ДАН СССР. 1983. Т. 270. №4. С. 781-785. [2] Дубровин Б. А., Новиков С. П. // УМН. 1989. Т. 44. №6. С. 29-98. [3] Уизем Дж. Линейные и нелинейные волны. М.: Мир, 1977. [4] Luke J. C. // Proc. Roy. Soc. London Ser. A. 1966. V. 292. № 1430. Р. 403-412. [5] Гринберг Н. И. // УМН. 1985. Т. 40. № 4. С. 217-218. [6] Новиков С. П., Мальцев А. Я. // УМН. 1993. Т. 48. № 1. С. 155-156. [7] Maltsev А. Yа. // solv-int/9611008, 1996 (to appear). 